\title{
Electrostatics of straight and bent nanotubes
}

\author{
E. G. Mishchenko and M. E. Raikh \\ Department of Physics, University of Utah, Salt Lake City, UT 84112
}

\begin{abstract}
Response of a single-walled carbon nanotube to external electric field, $F$, is calculated analytically within the classical electrostatics. Field-induced charge density distribution is approximately linear along the axis of a metallic nanotube and depends rather weakly, as $\ln (h / r)$, on the nanotube length, $h$, (here $r$ is the nanotube radius). In a semiconducting nanotube with a gap, $E_{g}$, charge separation occurs as $F$ exceeds the threshold value $F_{t h}=E_{g} / e h$. For $F>F_{t h}$, positively and negatively charged regions at the ends of nanotube are separated by a neutral strip in the middle. Properties of this neutral strip, length and induced charge distribution near the ends, are studied in detail. We also consider a bent nanotube and demonstrate that the number of neutral strips can be one or two depending on the direction of $F$.
\end{abstract}

PACS numbers: 71.20.Tx, 73.63.Fg, 78.67.Ch

\section{INTRODUCTION}

There are two types of devices based on carbon nanotubes (NTs) that are currently intensely investigated. These are field-effect transistors and field emitters for flat panel displays and x-ray sources. Although both types of devices were reported several years ago [1, 2] and significant improvement in their characterisitcs had been recently achieved (see e.g., Refs. [3, 4, 5] and Refs. [6, 7]), the understanding of performance of these devices is far from complete. The prime reason for this is 1D-like geometry of NT-based transistors and field emitters. Due to this geometry, their electrostatics is qualitatively different from that in respective well-understood 2D counterparts.

Traditionally, electrostatics for particular geometry of NT transistor [8, 9] or field emitter [10, 11] is studied theoretically by employing a certain version of first-principle calculations. Notable exceptions are Refs. 12, 13], in which classical electrostatics was used to describe the potential profile near the contact between metallic and semiconducting NTs as well as the contact between NT and a metal. Applicability of this description of the contact phenomena in NTs was later questioned in Ref. 14].

In general, it is not obvious whether or not, in terms of electrostatics, a NT of a small radius, $r$, can be modeled by an infinitely thin sheet of electron gas wrapped into a cylinder. In other words, whether or not the potential distribution can be adequately described by the Poisson equation with boundary conditions imposed at distance, $r$, from the NT axis. Positive answer to this question was recently given in Ref. [15], where the density-functional calculations of the extra charge distribution along the NT were shown to be in quantitative agreement with classical electrostatics analysis.

In this situation, it is instructive to consider a model problem of the classical electrostatics of a NT, which allows for an analytical solution. Qualitative features of this solution might then yield a valuable insight into electrostatics of realistic devices. Such a problem is studied in the present paper. Namely, we consider a NT in external electric field, $F$, parallel to the NT axis. Separation of variables in the Poisson equation in this geometry is impossible. Still, as we demonstrate below, presence of a small parameter, $r / h$, where $h$ is the NT length, allows one to obtain the asymptotically exact distribution of potential. We show that for metallic NT the density of induced charge changes linearly with distance from the NT center. For semiconducting NT with a gap, $E_{g}$, charge separation, which occurs as $F$ exceeds the threshold value $E_{g} / e h$, results in formation of a neutral strip with a width $\approx E_{g} / e F$ in the center of the NT. We find the profile of the charge density growth from the edges of the strip towards the NT ends. Finally, we use the developed approach to describe quantitatively the electrostatics of bent or wiggly NTs in external field, pertinent to recent electroabsorption measurements [16], and demonstrate that wiggling results in multiple alternating positively and negatively charged regions separated by neutral strips.

\section{BASIC EQUATION}

Denote with $\rho(z)$ the linear density of charge, induced by external field on the NT surface. Then the local value of the Fermi momentum is given by $p_{F}(z)=$ $\pi \hbar|\rho(z)| / 2 e N$, where $N$ accounts for the spin and band degeneracy $(N=2$ or 4 , and is determined by the NT chirality). The local chemical potential, $\mu(z)$, is related to $p_{F}(z)$ via the NT energy spectrum

$$
\mu(z)=\operatorname{sign}(z) \sqrt{E_{g}^{2} / 4+v_{0}^{2} p_{F}^{2}(z)},
$$

where $v_{0} \approx 8 \times 10^{7} \mathrm{~cm} / \mathrm{s}$ is the electron velocity in graphene. Second relation between $\mu(z)$ and $\rho(z)$ expresses the fact that the electrochemical potential remains constant along the nanotube, i.e. $\mu(z)+e \varphi(z)=0$, 
where $\varphi(z)$ is the electrostatic potential

$$
\varphi(z)=-F z+\frac{1}{e} \int_{-h / 2}^{h / 2} d z^{\prime} \rho\left(z^{\prime}\right) \Phi\left(z-z^{\prime}\right)
$$

which is created by external field and by induced charges. The kernel, $\Phi\left(z-z^{\prime}\right)$, in Eq. (2) takes a simple form in the case of isolated NT, lying on substrate with dielectric constant, $\varepsilon$

$$
\Phi(x)=\frac{e}{\pi \varepsilon^{*}} \int_{0}^{\pi} \frac{d \alpha}{\left[x^{2}+4 r^{2} \sin ^{2}(\alpha / 2)\right]^{1 / 2}},
$$

where $\varepsilon^{*}=(\varepsilon+1) / 2$. With the help of Eqs. (1) and (2), the condition of constant electrochemical potential can now be presented as a closed integral equation for $\rho(z)$

$$
e F z=\sqrt{\frac{E_{g}^{2}}{4}+\left[\frac{\pi \hbar v_{0} \rho(z)}{2 e N}\right]^{2}}+\int_{-h / 2}^{h / 2} d z^{\prime} \rho\left(z^{\prime}\right) \Phi\left(z-z^{\prime}\right),
$$

where we assumed $z$ to be positive. Eq. (4) should be complemented by the obvious condition that $\rho(z)$ is odd.

\section{ASYMPTOTIC SOLUTION OF EQ. (4)}

In order to make use of the small parameter $r / h$, we rewrite the integral on the rhs of Eq. (4) as follows

$$
\int_{-h / 2}^{h / 2} d z^{\prime} \rho\left(z^{\prime}\right) \Phi\left(z-z^{\prime}\right)=\int_{0}^{h / 2} d z^{\prime} \frac{\partial \rho\left(z^{\prime}\right)}{\partial z^{\prime}} K\left(z, z^{\prime}\right),
$$

where the function $K\left(z, z^{\prime}\right)$ is defined as

$$
K\left(z, z^{\prime}\right)=\int_{z^{\prime}}^{h / 2} d z_{1}\left[\Phi\left(z-z_{1}\right)-\Phi\left(z+z_{1}\right)\right] .
$$

Our most important observation is that, in the limit $h \gg r$, the function $K\left(z, z^{\prime}\right)$ can be replaced by $\left(2 e / \varepsilon^{*}\right) \ln (h / 4 r) \Theta\left(z-z^{\prime}\right)$, where $\Theta(x)$ is the stepfunction. Possibility of such a replacement is illustrated in Fig. 1. A simple from of $K\left(z, z^{\prime}\right)$ allows for a drastic simplification of Eq. (4), which transforms from intergral equation to a simple algebraic (quadratic) equation. In particular case, $E_{g}=0$ (metallic NT), we obtain the following result for the induced charge distribution

$$
\rho(z) \approx \frac{\varepsilon^{*} g F z}{1+2 g \ln (h / r)},
$$

where we have introduced a dimensionless interaction parameter $g=2 N e^{2} / \pi \varepsilon^{*} \hbar v_{0}$. The above result for $\rho(z)$ has a logarithmic accuracy, in the sense, that numerical factor in the argument of logarithm is not specified. In particular, the height of the step-function in Fig. 1 contains $1 / 4$ under the logarithm. Another contribution to the argument of logarithm comes from the smearing of the

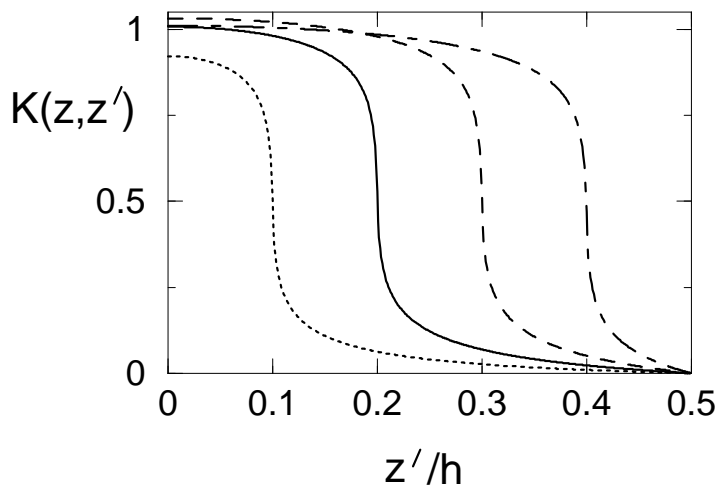

FIG. 1: Plot of the function $K\left(z, z^{\prime}\right)$ [scaled with the factor $\left.2 e \ln (h / 4 r) / \varepsilon^{*}\right]$ for $h / r=10^{3}$ and different values of $z: z=$ $0.1 h$ (dotted line), $z=0.2 h$ (solid line), $z=0.3 h$ (dashed line), $z=0.4 h$ (dash-dotted line).

step-function in Fig. 1. This smearing gives rise to the relative correction $-2 z \partial \ln \rho(z) / \partial z$ to $\ln (h / 4 r)$; in particular case of Eq. (7) this correction is equal to -2 . Overall, the condition of applicability of Eq. (7) is $\ln (h / r) \gg 1$, which is met in most of the realistic situations. It follows from Eq. (7) that the polarizability, $\chi$, of the NT, defined as $P(F)=\int_{-h / 2}^{h / 2} d z z \rho(z)=\chi F$, has the form

$$
\chi=\frac{\varepsilon^{*} g h^{3}}{12[1+2 g \ln (h / r)]} .
$$

It is clear from Eq. (8) that the product $g \ln (h / r) \approx$ $\left(1.74 N / \varepsilon^{*}\right) \ln (h / r)$ is a quantitative measure of the "metallicity" of the NT. In the limit of a "long" NT, when the product $g \ln (h / r)$ is large, we have $\chi=$ $\varepsilon^{*} h^{3} / 24 \ln (h / r)$, which coincides with the textbook expression 17] for polarizability of a perfectly conducting ellipsoid with axes $r$ and $h \gg r$. In this limit, with external field parallel to the NT axis, the resulting field at the NT surface is normal to this surface. The opposite limit, $2 g \ln (h / r) \ll 1$, of a "short" nanotube, when external field is altered weakly by the induced charges, cannot be achieved even for high dielectric constant of the substrate, e.g., $\varepsilon^{*} \approx 6$ for Si.

Consider now a semiconducting (or strained metallic [18]) NT with finite $E_{g}$. It is seen from Eq. (4) that charge separation occurs only when the external field is strong enough, namely, $F>F_{t h}=E_{g} / e h$. It also follows from Eq. (41) that, as $F$ increases, electrons and holes emerge at the NT ends, while the strip $|z|<E_{g} / 2 e F$ in the center of NT remains neutral. The behavior of $\rho(z)$ outside the strip is given by

$$
\begin{array}{r}
\rho(z)=\left(\frac{\varepsilon^{*} E_{g}}{2 e}\right)\left[g^{2} \ln ^{2}(h / r)-\frac{1}{4}\right]^{-1} \times \\
{\left[g^{2} \ln (h / r)\left(\frac{e F z}{E_{g}}\right)-\frac{g}{2} \sqrt{\left(\frac{e F z}{E_{g}}\right)^{2}+g^{2} \ln ^{2}(h / r)-\frac{1}{4}}\right]}
\end{array}
$$


and is illustrated in Fig. 2. From the edge of the neutral strip to the "bulk" of NT the slope of $\rho(z)$ decreases by a factor $2 g \ln (h / r) /[2 g \ln (h / r)+1]$. Using Eq. (9), one can calculate the induced dipole moment, $P(F)$, of semiconducting NT. Obviously, for $F \gg F_{t h}$ it is the same $\chi F$ as for metallic NT. In the vicinity of the threshold, $\left(F-F_{t h}\right) \ll F_{t h}$, the induced charge density not only occupies small region near the tips, but is also small in magnitude. Therefore, $P(F)$ is quadratic in $\left(F-F_{t h}\right)$ near threshold, namely

$$
P(F)=\frac{\varepsilon^{*} h^{3} F_{t h}}{4 \ln (h / r)}\left(\frac{F}{F_{t h}}-1\right)^{2} .
$$

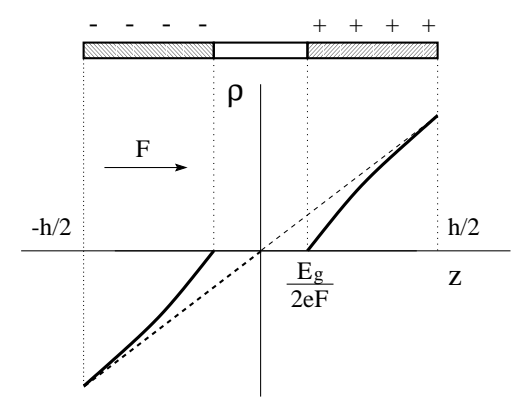

FIG. 2: Charge density distribution induced by external field, $F$, along the metallic (dashed line) and semiconducting (solid line) NT.

\section{SMALL $E_{g}$; FINE STRUCTURE OF THE NEUTRAL STRIP}

The boundaries of the neutral strip, $z_{t}= \pm E_{g} / 2 e F$, were found from Eq. (4) within the "local" approximation. There are two sources of corrections to this result: classical and quantum. Classical correction originates from the fact that for small $E_{g}$ the neutral strip is surrounded by long charged regions with opposite signs of charge. Setting $z=z_{t}$ in Eq. (4), and substituting into the rhs the zero-order result (9) for $\rho(z)$, we obtain the following modified equation for $z_{t}$

$$
z_{t}=\frac{E_{g}}{2 e F}+\frac{2 g \ln \left(h / z_{t}\right)}{1+2 g \ln (h / r)} z_{t} .
$$

The second term in the rhs of Eq. (11) reflects the fact that potentials, created by the left and right charged neighbors of the neutral strip, do not compensate each other completely. As follows from Eq. (11), the relative classical correction to the position of the boundary, $z_{t}$, is $\delta z_{t}^{c l} / z_{t} \approx 2 \ln \left(h / z_{t}\right) / \ln (h / r)$. This correction is small provided that $e F r \ll E_{g}$. The latter condition also insures that the underlying energy spectrum of the NT is not affected by $F$, as was assumed in derivation of our basic equation (4). Quantum correction to $z_{t}$ comes from the penetration of electronic wave functions into the classically forbidden region inside the neutral strip. Using Zener's formula for tunneling exponent in the Dirac spectrum (11), the relative quantum correction can be presented in the form, $\delta z_{t}^{q} / z_{t} \sim\left(l_{B} / g z_{t}\right)^{2 / 3}$, where $l_{B}=e^{2} / E_{g} \varepsilon^{*}$ has the meaning of the exciton Bohr radius. Thus, the condition of smallness of the quantum correction to $z_{t}$ coincides with the condition that $F$ is weaker than the ionization threshold for an exciton.

\section{$F=0 ;$ CHARGED METALLIC NT}

Experimental situations in which electrostatics of a charged NT is important are listed in Ref. 15]. Classical electrostatic analysis of a charged NT was carried out in this paper only for a short NT (with $h / r \approx 4$ ). Below we find the distribution of charge analytically in the limit $h \gg r$. For a charged metallic NT the condition of a constant electrochemical potential takes the form

$$
\mu=\left(\frac{\pi \hbar v_{0}}{2 e N}\right) \rho(z)+\int_{-h / 2}^{h / 2} d z^{\prime} \rho\left(z^{\prime}\right) \Phi\left(z-z^{\prime}\right) .
$$

Now $\rho(z)$ is even. To employ the above ansatz, we take derivative from the both sides of Eq. (12) and perform integration by parts in the rhs. This yields

$$
\rho(h / 2)\left[\Phi_{-}-\Phi_{+}\right]=\frac{e R(z)}{g \varepsilon^{*}}+\int_{-h / 2}^{h / 2} d z^{\prime} R\left(z^{\prime}\right) \Phi\left(z-z^{\prime}\right),
$$

where $R(z)=\partial \rho(z) / \partial z$ is the odd function of $z$, and a short-hand notation $\Phi_{ \pm}(z)=\Phi\left(\frac{h}{2} \pm z\right)$ is introduced. Now Eq. (13) has the form similar to Eq. (4). It should be complemented by the condition $2 \int_{0}^{h / 2} d z \rho(z)=Q$, where $Q$ is the total charge on the NT. Using the similarity between Eqs. (13) and (4), we readily obtain

$$
\rho(z)=\frac{Q}{h}\left[1+\frac{g}{1+g \ln (h / r)} \ln \left(\frac{h^{2}}{h^{2}-4 z^{2}}\right)\right] .
$$

Hence, $\rho(z)$ is essentially constant along the NT 19], and raises sharply only near the tips. This behavior compares favorably with numerical results [15]. Logarithmic divergence in Eq. (14) is terminated in the vicinity of the tip $h / 2-z \lesssim r$, so that the net growth of $\rho(z)$ is given by $\rho(h / 2) / \rho(0)=[1+2 g \ln (h / r)] /[1+g \ln (h / r)]$.

In optical experiments on separated NTs the tubes usually have wiggly shapes. Then their response to the external field can be quite peculiar, with numerous alternating positively and negatively charged regions separated by neutral strips (see Fig. 3a). An insight into electrostatics of a wiggly NT can be drawn from a model example that allows for exact solution. 


\section{BENT NT}

We consider a NT in the form of a semicircle of a radius, $R$. In two limiting cases, the electric field is either pointed along the diameter connecting the NT tips (parallel geometry) or perpendicular to this diameter (perpendicular geometry, see Fig. 3). Both geometries are

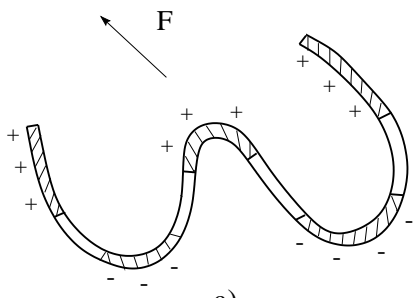

a)

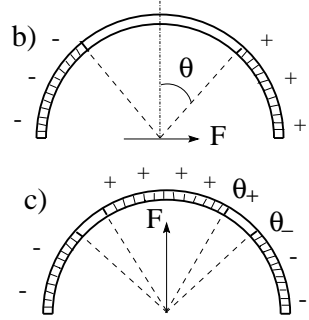

FIG. 3: Schematic illustration of charge separation in semiconducting NT in external field: (a) NT of a wiggly shape; (b) and (c) NT of a semicircle shape with $F$ parallel and perpendicular to the diameter, respectively.

described by Eq. (4) written in polar coordinates

$$
\begin{aligned}
& e F_{t}(\theta) R-\mathcal{C}=\operatorname{sign}(\rho) \sqrt{E_{g}^{2} / 4+\left[e \rho(\theta) / g \varepsilon^{*}\right]^{2}} \\
& +\frac{e R}{\varepsilon^{*}} \int_{-\pi / 2}^{\pi / 2} d \theta^{\prime} \rho\left(\theta^{\prime}\right) \Phi\left(R \sin \frac{\left|\theta-\theta^{\prime}\right|}{2}\right),
\end{aligned}
$$

where $F_{t}(\theta)$ is the tangent component of $F$. The constant, $\mathcal{C}$, which determines the electrochemical potential of NT, must be found from the condition of the net NT neutrality, $\int_{0}^{\pi} d \theta \rho(\theta)=0$. We emphasize that in both the parallel and perpendicular geometries only the tangent component of electric field is responsible for the charge separation. The normal component field has no effect on the charge separation if the condition $E_{g} \gg e F r$ is satisfied. The latter condition means that field-induced mixing of transverse subbands is negligible.

(i) In parallel geometry, we have $F_{t}=F \sin \theta$. Thus, $\rho(\theta)$ is odd, so that $\mathcal{C}=0$. Same ansatz as for a straight $\mathrm{NT}$, yields the following solution of Eq. (15) for $E_{g}=0$ $\rho(\theta)=\varepsilon^{*} g F R \sin \theta /[1+2 g \ln (R / r)]$. It is also easy to see from Eq. (15) that, for finite $E_{g}$, the neutral strip occupies the segment $|\theta|<\arcsin \left(2 e F R / E_{g}\right)$ of the NT near its top (Fig. 3b).

(ii) In perpendicular geometry, $F_{t}(\theta)=F \cos \theta$, is the even function of $\theta$. As a result, $\rho(\theta)$ differs qualitatively from the case of a straight NT. The form of $\rho(\theta)$ for this geometry can be found from the same ansatz Eq. (13) that was used for the straight charged NT. In particular, for $E_{g}=0$ we obtain

$$
\rho(\theta)=\varepsilon^{*} F R \frac{g(\cos \theta-2 / \pi)}{1+2 g \ln (R / r)} .
$$

The two points at which $\rho(\theta)$ changes the sign are therefore located at $\theta= \pm \theta_{0}= \pm 50.5^{\circ}$. For a finite $E_{g}$ two neutral strips are formed around $\theta= \pm \theta_{0}$. Their boundaries, $\pm \theta_{+}$and $\pm \theta_{-}$, (see Fig. $3 \mathrm{c}$ ) are determined by the conditions

$$
\cos \theta_{+}=\frac{2 \mathcal{C}+E_{g}}{2 e F R}, \quad \cos \theta_{-}=\frac{2 \mathcal{C}-E_{g}}{2 e F R} .
$$

When the gap is small, $E_{g} \ll e F R$, the centers of the strips are still located near $\pm \theta_{0}$, while the strip width, $\delta \theta=\left(\theta_{-}-\theta_{+}\right)$, can be found directly from Eq. (17), namely $\delta \theta=E_{g} / e F R \sin \theta_{0} \approx 1.3 E_{g} / e F R$.

For small ratio $E_{g} / 2 e F R$ it is also easy to trace the crossover between the parallel and perpendicular geometries as the field is rotated. For rotation angle, $\beta$, and for $E_{g}=0$, a straightforward generalization of Eq. (16) yields the following angular dependence of the charge density $\rho(\theta) \propto\left[\cos (\beta-\theta)-\frac{2}{\pi} \cos \beta\right]$. From this dependence we conclude that, as $\beta$ decreases from $90^{\circ}$ (parallel geometry), the narrow neutral strip at the top of semicircle moves to the left. At critical $\beta_{c}=\arctan (2 / \pi) \approx 32.5^{\circ}$, when the neutral strip is located around $\theta \approx 25^{\circ}$, the second neutral strip emerges at the right end of the NT. As $\beta$ decreases further, both neutral strips move to the left and assume their "perpendicular" positions $\theta= \pm 50.5^{\circ}$.

\section{EXPERIMENTAL IMPLICATIONS}

In Ref. 20 the potential distribution along the NT was measured using the atomic force microscope. Experimentally measured profile of the voltage drop in the structures with a small contact resistance is consistent with existence of a neutral strip near the NT center. Note, that the substrate in Ref. 20 was thick: $D=200$ or $D=500 \mathrm{~nm}$, but still thinner than the NT length, $h=1200 \mathrm{~nm}$. Taking into account the presence of a gate at distance $D \lesssim h$ from the NT amounts to replacement [21, 22, 23, 24] of $\ln (h / r)$ by $\ln (D / r)$ in all the above formulas.

Our results have direct relevance to the electro-optics of NTs. Measurements of electroabsorption in singlewalled carbon NTs were recently reported in Ref. 16. It might seem that with photon energy $\sim 1 \mathrm{eV}$ much bigger than $E_{g}$ the large-scale nanotube geometry is not important. This, however, is not the case. The reason is that the dipole moment of the many-body optical transition [25, 26] is directed along the tube [26, 27]. Even if external field is parallel to the tube axis, the resulting field is almost perpendicular to the nanotube surface, and thus its effect on the optical transitions is suppressed. This strong suppression by a factor $[1+2 g \ln (h / r)]$ must be taken into account when the oscillator strength is extracted from electroabsorption 16. On the other hand, within the neutral strip, the acting field is equal to the applied field. However, the relative contribution, $E_{g} / e F h$, 
of neutral strips to the absorption is small.

As a final remark, note that dimensionless parameter, $g$, which governs the screening properties of the NT, has a transparent meaning. For 2D electron gas with a density of states, $\nu$, the linear screening length is equal to $l=\varepsilon^{*} / 2 \pi \nu e^{2}$. If the gas is wrapped into a cylinder of a radius, $r$, then the degree of penetration of external field inside the cylinder is determined by the ratio $r / l$. Up to a numerical coefficient, this ratio is nothing but the parameter $g$.

\section{ACKNOWLEDGEMENTS}

This work was supported by NSF under Grant No. DMR-0503172 and by the Petroleum Research Fund under Grant No. 43966-AC10. Useful discussions with Z. V. Vardeny on optics of NTs are acknowledged.

[1] S. J. Tans, A. R. M Verschueren, and C. Dekker, Nature (London) 393, 49 (1998).

[2] A. G. Rinzler, J. H. Hafner, P. Nikolaev, L. Lou, S. G. Kim, D. Tomanek, P. Nordlander, D. T. Colbert, and R. E. Smalley, Science 269, 1550 (1995).

[3] A. Javey, J. Guo, Q. Wang, M. Lundstrom, and H. Dai, Nature 424, 654 (2003).

[4] X. Zhou, J-Y. Park, S. Huang, J. Liu, P. L. McEuen, Phys. Rev. Lett. 95, 146805 (2005).

[5] W. Kim, A. Javey, R. Tu, J. C. Cao, Q. Wang, and H. Dai, Appl. Phys. Lett. 87, 173101 (2005), and references therein.

[6] S. Fan, M. G. Chapline, N. R. Franklin, T. W. Tombler, A. M. Cassell, and H. Dai, Science, 283, 512 (1999).

[7] M. Mauger, Vu Thien Binh, A. Levesque, and D. Guillot, Appl. Phys. Lett. 85, 305 (2004).

[8] Y. Xue and S. Datta, Phys. Rev. Lett. 83, 4844 (1999).

[9] Y. Xue and M. A. Ratner, Phys. Rev. B 69, 161402(R) (2004).
[10] A. Mayer, N. M. Miskovsky, and P. H. Cutler, Phys. Rev. B 65, 155420 (2002).

[11] A. Buldum and J. P. Lu, Phys. Rev. Lett. 91, 236801 (2003).

[12] A. A. Odintsov, Phys. Rev. Lett. 85, 150 (2000).

[13] A. A. Odintsov and Y. Tokura, J. Low Temp. Phys. 118, 509 (2000).

[14] F. Léonard and J. Tersoff, Phys. Rev. Lett. 84, 4693 (2000); ibid. 85, 4767 (2000).

[15] P. Keblinski, S. K. Nayak, P. Zapol, and P. M. Ajayan, Phys. Rev. Lett. 89, 255503 (2002).

[16] J. W. Kennedy, Z. V. Vardeny, S. Collins, R. H. Baughman, H. Zhao, S. Mazumdar, preprint cond-mat/0505071

[17] L. D. Landau and E. M. Lifshitz, Electrodynamics of Continuous Media (Pergamon Press, Oxford, 1984).

[18] E. D. Minot, Y. Yaish, V. Sazonova, J.-Y. Park, M. Brink, and P. L. McEuen, Phys. Rev. Lett. 90, 156401 (2003).

[19] charge distribution was studied by means of electrostatic force microscopy in M. Paillet, P. Poncharal, and A. Zahab, Phys. Rev. Lett. 94, 186801 (2005).

[20] Y. Yaish, J.-Y. Park, S. Rosenblatt, V. Sazonova, M. Brink, and P. L. McEuen, Phys. Rev. Lett. 92, 046401 (2004).

[21] K. A. Bulashevich and S. V. Rotkin, JETP Lett. 75, 205 (2002).

[22] S. Sapmaz, Ya. M. Blanter, L. Gurevich, and H. S. J. van der Zant, Phys. Rev. B 67, 235414 (2003).

[23] L. Latessa, A. Pecchia, A. Di Carlo, and P. Lugli, Phys. Rev. B 72, 035455 (2005).

[24] M. P. Anantram and F. Léonard Rep. Prog. Phys. 69, 507 (2006), and references therein.

[25] C. L. Kane and E. J. Mele, Phys. Rev. Lett. 90, 207401 (2003); 93, 197402 (2004); C. D. Spataru, S. Ismail-Beigi, L. X. Benedict, and S. G. Louie, ibid. 92, 077402 (2004); V. Perebeinos, J. Tersoff, and P. Avouris. ibid, 92, 257402 (2004).

[26] H. Zhao and S. Mazumdar, Phys. Rev. Lett. 93, 157402 (2004).

[27] W.-Z. Liang G.-H. Chen, Z. Li, and Z.-K. Tang, Appl. Phys. Lett. 80, 3415 (2002). 